\title{
ESTRATÉGIAS UTILIZADAS PELAS ENFERMEIRAS NA ATENÇÃO BÁSICA PARA A PREVENÇÃO DA GRAVIDEZ NA ADOLESCÊNCIA
}

\section{STRATEGIES USED BY NURSES IN BASIC CARE UNITS FOR THE PREVENTION OF TEENAGE PREGNANCY}

\author{
Raphael Silva Nogueira Costa ${ }^{1}$ \\ lara Pereira Fonseca ${ }^{2}$ \\ Fábio Lisboa Barreto ${ }^{3}$ \\ Maria Talita Cruz Silva Oliveira ${ }^{4}$ \\ Michelle Viçoso Gomes Lopes ${ }^{5}$
}

\begin{abstract}
A cada dia, mais precocemente, os adolescentes vêm iniciando suas atividades sexuais, o que pode levar a uma gravidez não planejada. Este estudo teve como objetivo analisar as estratégias desenvolvidas por enfermeiras da atenção básica quanto à prevenção da gravidez na adolescência em um município do Recôncavo da Bahia. Tratase de um estudo descritivo com abordagem qualitativa, realizado com 15 enfermeiras atuantes na atenção básica do município. A técnica de coleta de dados foi a entrevista individual semi-estruturada. Os dados foram analisados através da análise de conteúdo de Minayo. O resultado do estudo mostrou que as principais estratégias para prevenção da gravidez na adolescência é a dispensação de métodos contraceptivos, o que põe em evidência a falta de ações como sala de espera, palestras e atividades de sensibilização individuais no cotidiano da atenção básica. A captação de adolescentes para consultas ocorre na sua maior parte por busca ativa com os agentes comunitários de saúde. Como barreiras foram citadas pelas enfermeiras a vergonha das adolescentes de irem à unidade no dia de atendimento de Planejamento Familiar, a necessidade de autorização dos pais para frequentarem as consultas e a não participação masculina nesse processo. Foram mencionadas também estratégias como as ações intersetoriais, através do programa Saúde na Escola, porém estas não ocorrem de maneira contínua. Com este estudo, percebeu-se que as ações de prevenção da gravidez realizadas pelas enfermeiras da atenção básica encontra entraves para sua efetiva concretização, sendo centradas ainda na entrega de métodos contraceptivos e se afastando do princípio da integralidade da atenção.
\end{abstract}

Palavras-chave:Adolescente. Enfermagem em Saúde Comunitária.Planejamento Reprodutivo.

Every day, more precociously, teenagers start their sexual activities, which may result in unplanned pregnancies. This study aimed to analyze the strategies developed by basic care nurses for the prevention of teenage pregnancy in a municipality of Recôncavo da Bahia. This is a descriptive study with a qualitative approach, carried out with 15 nurses in primary health care units. Data collection was carried out by means of semi-structured interviews and the analysis was performed using Minayo's content analysis. The result of the study showed that the main strategies used by the nurses was the distribution of contraception, which reveals the lack of actions such as waiting room conversations, lectures and individual awareness activities. Teen recruitment for consultation is carried out by community health agents through active search. Barriers mentioned by nurses included teenagers' shame to visit the unit for family planning, parents' consent to visit the doctor and the absence of male participation in the process. The nurses also mentioned the Intersectoral Health Program at school that should occur continuously, but does not. This study shows that the pregnancy prevention actions carried out by nurses at basic attention units encounters obstacles for effective implementation, is confined to the dispensation of birth control methods and thus moving away from the principle of comprehensive care.

Keywords: Teenagers. Community Health Nursing. Reproductive Planning.

'Enfermeiro, Mestre em Saúde Coletiva, Residente em Enfermagem Obstétrica pela Universidade Federal da Bahia (UFBA) - Salvador /Bahia. CV: http://lattes.cnpq.br/5314273426058220 E-mail:raphaelsnc@gmail.com.

${ }^{2}$ Enfermeira, Graduanda pela Faculdade Maria Milza (FAMAM) - Governador Mangabeira/Bahia. CV: http://lattes.cnpq.br/1257426555019244 E-mail: iarinhapereira@hotmail.com.

${ }^{3}$ Enfermeiro, Especialista em Auditoria de Serviços e Sistemas de Saúde, Docente da Faculdade Maria Milza (FAMAM) - Governador Mangabeira/Bahia. CV: http://lattes.cnpq.br/46274444855745152. E-mail: lisboa.auditor@gmail.com.

${ }^{4}$ Discente do Bacharelado de Enfermagem na Faculdade Maria Milza (FAMAM) - Governador Mangabeira/Bahia. CV: http://lattes.cnpq.br/5593049630015985. E-mail: tali oliveira@outlook.com.

5 Enfermeira, Residente em Enfermagem Ob̄stétrica pela Universidade Federal da Bahia (UFBA) - Salvador /Bahia. CV: http://lattes.cnpq.br/092321919843280.E-mail: milopes123@yahoo.com.br. 


\section{INTRODUÇÃO}

A adolescência é uma etapa de crescimento e desenvolvimento do ser humano, na qual ocorrem transformações corporais, hormonais e até mesmo comportamentais. É a fase em que acontece a transição entre a infância e a idade adulta. A lei brasileira, através do Estatuto da Criança e do Adolescente (ECA), considera adolescente o indivíduo entre 12 e 18 anos (BRASIL, 2007).

As dificuldades do acesso a informações educativas pode gerar impactos importantes, principalmente na adolescência, a exemplo de gravidez precoce, infecções sexualmente transmissíveis (IST) e AIDS. Esses impactos são maiores quanto menor for a idade da adolescente (COSTA; QUEIROZ; ZEITOUNE, 2012).

No que se refere à falta de informação sobre contracepção, observa-se que o início do uso de contraceptivos ocorre de forma tardia e, muitas vezes, inadequada. O desconhecimento das funções do próprio corpo quanto à reprodução contribui para que ocorra atividade sexual desprotegida e despreocupada (TABORDA et al., 2014).

Por esses motivos, aumenta a possibilidade da ocorrência de uma gestação, que normalmente acarreta mudanças significativas no estilo de vida da mulher, e quando acontece na adolescência, surgem riscos adicionais, devido às mudanças físicas que ocorrem na criança ao tornar-se adulta. Por isso, quando uma adolescente engravida, os impactos biológicos, psicológicos e sociais são ainda maiores (FONSECA, 2004).

Nesse sentido, a mudança do corpo gera sentimentos contraditórios nas gestantes adolescentes, que podem Ihe causar ansiedade e desejo de retornar ao seu corpo antes do estado gravídico, fazendo-a se sentir envergonhada, insatisfeita e retraída (ALVES; ALBINO; ZAMPIERI, 2011).

Assim, toda gravidez que não é planejada pode resultar em impactos psíquicos e emocionais negativos, principalmente na adolescência, pois leva a mudanças nos planos de vida para o futuro, dependência financeira, além da perda de liberdade, fatores que podem alterar o seu estado emocional (MANFRÉ; QUEIRÓZ; MATTHES, 2010).

A gravidez não planejada na adolescência, por ser considerado um problema de saúde pública, exige programas de orientação, preparação e acompanhamento durante a gravidez e o parto, para disminuir os riscos durante o desenvolvimento da criança e para garantir a saúde da própria gestante (ARAÚJO et al., 2015). Nesse contexto, as enfermeiras possuem um papel essencial na prevenção da gravidez na adolescência, através de ações preventivas e educativas que promovam a saúde reprodutiva.

O planejamento reprodutivo (PR) é um programa do Ministério da Saúde para o desenvolvimento de ações que auxiliem as pessoas que pretendem ter filhos ou que preferem adiar o crescimento da família. Estas ações estão drecionadas a indivíduos em diversas situações, desde relação conjugal estável, vida sexual sem parceiros estáveis e aqueles que planejam iniciar sua vida sexual. A assistência ao $\mathrm{PR}$, atualmente, é realizada pelas unidades de saúde da Atenção Básica, que devem ter uma disponibilidade diversificada de insumos e tecnologias conceptivas e contraceptivas (SILVA; CARVALHO, 2007).

O objetivo desta pesquisa é analisar as estratégias desenvolvidas por enfermeiras das unidades de Saúde da Família quanto à prevenção da gravidez na adolescência de um município do Recôncavo da Bahia.

\section{METODOLOGIA}

Trata-se de um estudo descritivo com abordagem qualitativa, que foi desenvolvido nas 15 unidades de Saúde da Família (USF) da Atenção Básica de um município do Recôncavo da Bahia.

As participantes foram enfermeiras que estavam atuando em todas as USF do município, sendo 11 na zona urbana e 4 na zona rural. Os critérios de inclusão para participar do estudo foram ser enfermeira 
com, pelo menos, três meses de atuação em USF; estar presente no dia da coleta de dados e aceitar assinar o Termo de Consentimento Livre e Esclarecido (TCLE). Desta forma, quanto aos critérios de exclusão, seriam excluídas do estudo as enfermeiras que estivessem de férias ou afastadas por outros motivos ou as que não aceitassem participar da pesquisa.

O presente estudo foi aprovado pelo Comitê de Ética em Pesquisa da Faculdade Maria Milza sob parecer de número 2.252.612 de 31 de agosto de 2017, atendendo desta forma os preceitos éticos dispostos na Resolução n 466 de 12 de dezembro de 2012 do Conselho Nacional de Saúde (CNS).

Como técnicas de coleta de dados utilizou-se a entrevista com roteiro semiestruturado, que foi realizada em local reservado nas próprias USF onde atuavam as enfermeiras, objetivando entender como se dá a sua atuação na USF quanto à prevenção da gravidez na adolescência.

De posse dos dados coletados, foi realizada uma pré-leitura, para ordenação dos mesmos e após leitura exaustiva, os dados foram classificados e distribuídos em categorias para posterior análise. Assim, tendo como base os objetivos deste estudo, foi utilizada a análise temática de conteúdo de Minayo (2006).

\section{RESULTADOS E DISCUSSÃO}

\section{CARACTERIZAÇÃO DAS PARTICIPANTES}

As enfermeira participantes deste estudo estavam na faixa etária de 23 a 60 anos (média 33,6), com tempo de formação que variava de 7 meses a 30 anos (média de 6 anos). 0 tempo de atuação no município da maioria das entrevistadas foi de menos de 1 ano, com a média de 7 meses e meio; apenas uma entrevistada atuava no município há mais de 20 anos. Quanto à formação, oito tinham finalizado cursos de especialização e outras cinco ainda não os tinham concluido. No que diz respeito à temática dos cursos, 6 enfermeiras relataram ser na área de Saúde Coletiva em PSF, ESF ou Atenção Básica, e 9 em outras aéreas no campo da Saúde (Quadro 1).

Quadro 1. Caracterização das enfermeiras que atuavam na Atenção Básica de um município do Recôncavo da Bahia no ano de $2017(\mathrm{~N}=15)$.

\begin{tabular}{|l|l|l|l|l|l|l|}
\hline Entrevistada & $\begin{array}{c}\text { Localização } \\
\text { da unidade }\end{array}$ & \multicolumn{1}{|c|}{ Sexo } & \multicolumn{1}{|c|}{ Idade } & $\begin{array}{l}\text { Tempo de } \\
\text { formação }\end{array}$ & $\begin{array}{c}\text { Tempo de } \\
\text { atuação }\end{array}$ & Pós-graduação \\
\hline Enf1 & Zona rural & Feminino & 31 anos & 8 anos & 8 mêses & Sim \\
\hline Enf2 & Zona rural & Feminino & 29 anos & 7 anos & 9 meses & Sim \\
\hline Enf3 & Zona urbana & Feminino & 29 anos & 7 anos & 7 meses & Sim \\
\hline Enf4 & Zona urbana & Feminino & 28 anos & 2 anos & 6 meses & Não \\
\hline Enf5 & Zona urbana & Feminino & 33 anos & 5 anos & 6 meses & Sim \\
\hline Enf6 & Zona rural & Feminino & 27 anos & 8 meses & 8 meses & Em andamento \\
\hline Enf7 & Zona urbana & Feminino & 31 anos & 5 anos & 8 meses & Não \\
\hline Enf8 & Zona rural & Feminino & 24 anos & $\begin{array}{l}1 \text { anos e } 7 \\
\text { meses }\end{array}$ & 8 meses & Em andamento \\
\hline Enf9 & Zona urbana & Feminino & 23 anos & 7 meses & 7 meses & Em andamento \\
\hline Enf10 & Zona urbana & Feminino & 28 anos & 5 anos & 8 meses & Sim \\
\hline Enf11 & Zona urbana & Feminino & 43 & 7 anos & 8 meses & Sim \\
\hline Enf12 & Zona urbana & Feminino & 35 anos & 5 anos & 7 meses & Em andamento \\
\hline Enf13 & Zona urbana & Feminino & 28 anos & 5 anos & 8 meses & Em andamento \\
\hline Enf14 & Zona urbana & Feminino & 60 anos & 3 anos & 8 meses & Sim \\
\hline Enf15 & Zona rural & Feminino & 56 anos & 30 anos & 20 anos & Sim \\
\hline
\end{tabular}

Fonte: Dados da pesquisa, 2017. 


\section{ESTRATÉGIAS UTILIZADAS PELAS ENFERMEIRAS PARA ATIVIDADES DE PREVENÇÃO DA GRAVIDEZ NAADOLESCÊNCIA}

Esta categoria de análise apresenta e discute as estratégias utilizadas pelas enfermeiras para atividades de prevenção da gravidez na adolescência na USF.

Das entrevistadas, 15 referiram usar como estratégias para prevenção da gravidez na adolescência a distribuição de métodos contraceptivos, com ênfase nas falas abaixo:

As estratégias que utilizo são dispensação de métodos de barreiras além dos métodos contraceptivos (Enf1).

Orientação, a gente explica os métodos que temos disponíveis e explica o mais aconselhável pra ela estar utilizando naquele momento, oriento também sobre a importância do PF não apenas para prevenir gravidez, mas para evitar doenças (Enf7).

Durante a consulta acolho a adolescente da melhor forma possível, faço um bate papo sobre coisas da vida, para poder criar uma intimidade com elas, apresento os métodos, explico cada um deles, as reações adversas que pode ter, o que pode acontecer durante o ciclo , o período do retorno e aí a gente decide juntas qual o método mais indicado pra ela e dou orientação em relação ao preservativo que o método vai ajudar com a prevenção da gestação, mas quanto a prevenir doenças, o método não funciona dessa forma. Ela já sai daqui com a consulta agendada (Enf8).

Qualquer adolescente que vem até a unidade tomar a vacina eu sempre chamo para conversar, pergunto se já está tendo relação, se tem interesse em fazer algum tipo de PF. Explico direitinho a função do PF, que muita gente pensa que é só para proteger de engravidar, mas é para pessoas que pretendem ter filhos também. Eu tenho esse mural aqui na parede com todos os métodos, explico método por método, qual é melhor para elas poderem visualizarem, explico qual a menstruação desce qual não desce, explico a importância do preservativo, que mesmo tomando medicamento a camisinha previne doenças e deixo elas à-vontade para escolher os métodos, exceto a de 15 anos se escolher a injetável de 3 meses explico que naquele momento não é ideal para ela (Enf9).

Observa-se nos discursos das enfermeiras que as principais estratégias utilizadas por elas são a dispensação dos métodos contraceptivos, sendo que existem outras ações para o planejamento familiar, quando realizado sob a ótica da integralidade. Entre elas, podemos citar a sala de espera, o Programa Saúde na Escola, educação em saúde individual e coletiva, orientação aos pais em relação à importância da filha(o) ir até a unidade participar das ações e se prevenir. Essa falta de estratégias também foi diagnosticada nas observações de campo durante o atendimento com as adolescentes.

Diante disso, Higarashie et al.(2011) explicam que as enfermeiras que atuam na atenção básica devem entender que a integralidade do atendimento aos adolescentes é primordial para que se tenha uma atenção à saúde sexual e reprodutiva de qualidade, voltada para este grupo etário. Porém, esta integralidade, na maioria das vezes, não é colocada em prática, principalmente devido à falta de recursos e materiais, às dificuldades para disseminar informações, carência de insumos básicos, pouca aceitação da população-alvo em participar das atividades propostas pela equipe, à burocratização do sistema e à falta de tempo dos profissionais envolvidos. Neste sentido, a consulta de enfermagem do planejamento reprodutivo fica prejudicada, restringindo-se apenas aos casos em que o adolescente procura a unidade por iniciativa própria.

O Ministério da Saúde aponta que o planejamento familiar é um conjunto de ações em que são oferecidos todos os recursos para concepção e anticoncepção, e tem como objetivos ampliar e qualificar a atenção de planejamento familiar, incluindo a assistência à infertilidade; garantir a oferta de métodos- 
anticoncepcionais para a população em idade reprodutiva; ampliar o acesso de mulheres às informações sobre as opções de métodos anticoncepcionais e estimular a participação e a inclusão de homens, mulheres e adolescentes nas ações de planejamento familiar (BRASIL, 2006).

Quanto aos métodos contraceptivos oferecidos pelo Ministério da Saúde, os adolescentes podem optar por quase todos, menos os cirúrgicos, que devem obedecer alguns critérios para serem realizados e a injeção trimestral e as minipílulas, que não devem ser usadas antes dos 16 anos. No entanto, alguns métodos são mais adequados para esta fase da vida como, por exemplo, o preservativo masculino ou feminino (BRASIL, 2006).

O documento supracitado ressalta também que a camisinha masculina ou feminina deve ser usada em todas as relações sexuais, independentemente do uso de outro método anticoncepcional, pois a camisinha é o único método que oferece dupla proteção, ou seja, protege ao mesmo tempo das DST, Aids e da gravidez indesejada.

\section{DIFICULDADES E BARREIRAS PARAPREVENÇÃO DA GRAVIDEZ NA ADOLESCÊNCIA}

As enfermeiras, ao serem questionadas sobre as dificuldades e barreiras enfrentadas no contato com as adolescentes, explicaram que o sentimento de vergonha das adolescentes dificulta o seu trabalho, como pode ser constatado nas falas abaixo:

Sim, tenho dificuldades, pois acho que as jovens têm vergonha de vir até a unidade, os pais não têm essa orientação com os filhos, por isso elas não vêm. Vergonha que os pais saibam que já estão tendo relação. Quando vem já estão gestantes (Enf3).

As adolescentes têm vergonha de procurar a unidade de saúde porque elas acham que as pessoas vão saber que elas estão fazendo o PF, vão falar que estão tendo relações. Principalmente, na zona rural que as pessoas são cheias de princípios (Enf15).

Verifica-se como dificuldades e barreiras relatadas pelas enfermeiras que as jovens sentem não só vergonha, mas também medo das pessoas saberem que elas iniciaram a sua vida sexual.

É importante mencionar que alguns pais não autorizam que as filhas frequentem o atendimento de planejamento familiar. Contudo, a orientação às adolescentes e aos pais frente a essa temática deve ser estabelecida através de ações educativas, sobretudo, para que entendam que a assistência de enfermagem se faz sob sigilo e que o principal objetivo do atendimento para a jovem é a prevenção da gravidez na adolescência.

Foi possível perceber nas observações de campo, durante o atendimento com as adolescentes, quando era primeira consulta e a enfermeira perguntava se os pais sabiam que elas estavam lá, as mesmas diziam que não. Nesses casos, algumas enfermeiras não iniciavam a ação de prevenção de gravidez, mas solicitavam que elas retornassem com a autorização dos pais. Isso significa que, se esses pais não autorizarem a consulta, essa jovem corre o risco de adotar um método contraceptivo de maneira inadequada ou não fazer uso dele.

Estudos apontam que os enfermeiros encontram algumas dificuldades quanto ao desenvolvimento das ações preventivas da gravidez na adolescência. Entre elas, destacam-se a falta de adesão dos adolescentes; falta de capacitação dos profissionais na saúde de adolescentes; falta de infra-estrutura; falta de planejamento; falta de colaboração da direção das escolas e excesso de trabalho. Outro fator que dificulta o trabalho do enfermeiro é a falta de comunicação entre as equipes e vergonha das adolescentes em relação aos pais e a comunidade (RIBEIRO et al., 2016).

Outros problemas enfrentados pelos profissionais de saúde incluem a falta de capacitação para lidar com as adolescentes, de forma a poder criar estratégias que estimulem os jovens a procurar a unidade de saúde, pois muitos não se sentem bem acolhidos (RIBEIRO et al., 2016).

A comunicação entre e o enfermeiro e o adolescente é muito importante, para criar um vínculo de 
respeito e confiança. Para que isso aconteça, é necessário que todos os membros da equipe estejam aptos para prestar assistência adequada, com recursos materiais adequados e estrutura para que possa ser desenvolvido um atendimento qualificado (HIGARASHI et al., 2011).

Algumas enfermeiras referem dificuldades, no que diz respeito às adolescentes não retornarem na data marcada.

\begin{abstract}
Sim, encontro dificuldades em relação à adesão ao programa e voltar no dia marcado para o retorno. Como são menores de idade, não podemos fazer a medicação sem a presença de um responsável só para não correr o risco de vir uma gestação depois (Enf11).
\end{abstract}

Sim, a dificuldade maior é com relação aos retornos delas para dar continuidade ao método, elas não retornaram no período que agente marca e se é uma injetável mensal é para retornar em um dia especifico, contamos 30 dias, as vezes elas não retornam ou retornam com atraso (Enf7).

Verifica-se que entre as dificuldades e barreiras encontradas pelos profissionais está o fato das jovens não retornarem à unidade na data marcada, o que aumenta o risco de gravidez, pois todo método, seja oral ou injetável, têm data de início e término. Foi possível observar, durante o atendimento às adolescentes, que as enfermeiras orientam sobre a importância das jovens retornarem na data marcada.

A falta de interesse dos adolescentes em procurar o serviço e a falta de comprometimento em retornar na data marcada para dar continuidade, dificulta a captação e as estratégias criadas para prevenção da gravidez. Higarashi et al. (2011) enfatizam a importância de que todos os membros da equipe estejam integrados e capacitados para prestar a assistência adequada aos adolescentes, além de contar com recursos materiais e estrutura adequados para que possa ser desenvolvido um atendimento qualificado.

No entanto,quatro enfermeiras relataram não encontrar esse tipo de dificultades:

Não encontro dificuldades porque a cabeça dos adolescentes está bem diferente de antigamente, eles são muito mais abertos, eles procuram muito a unidade (Enf4)

É importante ressaltar que no caso dessas quatro profissionais, a unidade na qual trabalham está localizada na zona urbana e o perfil das usuárias é diferente, pois as jovens estão mais informadas por terem acesso a internet e procuram a unidade por medo de engravidar. A visita espontânea das jovens à unidade facilita o trabalho das enfermeiras no que diz respeito ao trabalho de orientação e prevenção de DST e gravidez indesejada.

Estudos apontam que $87,8 \%$ dos adolescentes brasileiros estão recebendo mais informações em relação à prevenção de DST e gravidez não planejada e $79,2 \%$ sabem como evitar gravidez. Mais da metade $(68,4 \%)$ dos entrevistados também sabia que era possível adquirir preservativos gratuitamente no Sistema Único de Saúde (SUS). O estudo ainda mostra que $27,5 \%$ dos jovens já iniciaram a vida sexual e que 66,2\% utiliza preservativos (BRASIL, 2016).

\title{
CAPTAÇÃO DAS ADOLESCENTES PARA ATIVIDADE DE PREVENÇÃO DA GRAVIDEZ NA ADOLESCÊNCIA
}

Esta categoria de análise apresenta e discute a atuação das enfermeiras frente à captação das adolescentes para atividade de prevenção da gravidez na adolescência naAtenção Básica de Saúde.

Dentre as entrevistadas, 14 enfermeiras relataram que a captação das adolescentes acontece 
através da busca ativa realizada pelos agentes comunitários de saúde ou de outros profissionais.

Junto com ACS fazemos um levantamento do quantitativo de adolescentes para fazer as palestras educativas para prevenção da gravidez, nas escolas enfatizam sobre os contraceptivos principalmente em épocas festivas. Existe o apoio dos profissionais das escolas (Enf3).

A gente faz busca ativa e os ACS ajudam diretamente nessa busca (Enf4).

Busca ativa, os ACS ajudam muito pelo fato deles conhecerem a comunidade. Principalmente as meninas vulneráveis, que tem relação sexual eles encaminham pra aqui. Nas escolas, a gente vai começar até fazer atividade sobre gravidez na adolescência e IST (Enf5).

A captação é com ajuda dos ACS, fazem busca ativa, visitando e convidando esses jovens para ir até a unidade (Enf6).

A captação das adolescentes para atividade de prevenção de gravidez na adolescência, como informam as enfermeiras, é realizada pelos agentes comunitários de saúde, o que mostra a importância do trabalho em equipe.

Porém, uma enfermeira relatou que a captação acontece através de conversas informais sobre as atividades realizadas, sala de espera e PSE criando um elo entre a unidade de saúde e a escola.

A captação é através de uma estar falando com a outra e também eu indo até as escolas fazer palestras,falo do programa. Os ACS não captam muito, porque eles se sentem constrangidos de chegar até eles porque os adolescentes são muito difíceis de estar lidando. Se você vai atrás deles querendo mandar eles ir até a unidade fazer o PF, eles vão dizer que direito você tem de estar interferindo na minha vida, então os ACS ficam muito apreensivos (Enf15).

Conforme a fala de Enf15, as opiniões sobre o atendimento nas unidades básicas transmitidas pelos frequentadores em conversas informais com membros da sua comunidade têm uma influência maior que a captação realizada pelos ACS, podendo ser negativas ou positivas. Os comentários positivos estimulam as adolescentes a frequentar as unidades de Atenção Básica e é importante, nesses casos, que as adolescentes sejam bem acolhidas pelo pessoal responsável para convencê-las a participar dos programas de planejamento familiar.

O papel da Enfermagem é de fundamental importância na orientação das adolescentes para identificar seus problemas, evitar uma gravidez indesejada e informar sobre os riscos das IST. No entanto, isso não diminui a importância dos ACS, pois eles são educadores de saúde, oganizam o acesso, captam necessidades, identificam prioridades, detectam os casos de risco e fazem busca ativa (SILVA, 2002).

As ações de promoção da saúde permeiam a consulta de enfermagem e as atividades em grupo, ressaltando o acolhimento, escuta qualificada e a ética como dispositivos que contribuem para promover um ambiente favorável à saúde do adolescente. Recomenda-se que as diretrizes propostas pelas políticas de saúde do adolescente sejam fortalecidas, no âmbito municipal, de forma que proporcionem à enfermeira condições de promover ações intersetoriais e interdisciplinares de educação sexual, na perspectiva de prevenção da gravidez precoce, que integrem família, escola, e comunidade, contribuindo para o exercício de uma sexualidade mais responsável e segura (GURGEL et al., 2008).

A enfermagem vem demonstrando empenho na medida em que se volta à construção de novas políticas e práticas em saúde, propondo medidas para a saúde individual e comunitária dos adolescentes 
e a incorporação de novas tecnologias educacionais e assistenciais que promovem a saúde desse grupo. Por meio de palestras educativas nas escolas e nas unidades de saúde e da sensibilização da equipe multiprofissional, é possível fortalecer o vínculo entre o enfermeiro e o adolescente para um atendimento integral e individual, através da consulta de enfermagem (RIBEIRO et al., 2016).

Conforme Almeida e Centa (2008), a enfermeira, enquanto profissional capacitada para assistir ao indivíduo em todas as etapas de vida, necessita estar inserida no Programa de Educação Sexual das escolas, promovendo ações e programas voltados para a saúde do adolescente e sua família, que devem atender as reais necessidade de ambos. É fundamental que tanto o governo, quanto a família, a escola e a sociedade não economizem esforços para promover a sexualidade com responsabilidade.

A orientação sexual de adolescentes é um assunto bastante abordado na atualidade, mas no ambiente escolar não é um assunto fácil de ser trabalhado, pois envolve vários protagonistas: a escola, os educadores, a família, a enfermagem, uma equipe multidisciplinar e o próprio adolescente. O trabalho de orientação do enfermeiro pode ser realizado junto ao adolescente, levantando diretamente com eles suas necessidades de conhecimento ou formando multiplicadores, que de acordo com trabalhos existentes, demonstram sucesso, pois o adolescente bem informado pode também transmitir conhecimento informalmente (COSTA; QUEIROZ; ZEITOUNE, 2012).

Embora a educação sexual esteja prevista no currículo escolar, ela precisa ser implementada de forma eficaz dentro das escolas, que podem inclui-la no seu projeto pedagógico, aproveintando o interesse que o tema desperta nessa etapa da vida e sua importância na construção da identidade de cada indivíduo.

Prado (2001) ressalta que as atividade escolares voltadas para a educação sexual ganham evidência quando se observa que a educação pública é responsável por 80 a $90 \%$ dos alunos do Brasil. Esse dado mostra o impacto que a educação sexual pode ter para promover a saúde e a sexualidade responsável, assim como prevenir a transmissçao de IST, HIVIAIDS e o uso de drogas entre adolescentes.

\section{AUSÊNCIAMASCULINANO PLANEJAMENTO REPRODUTIVO}

Nesta seção apresentamos e discutimos a não participação masculina nas questões do planejamento reprodutivo. Os adolescentes do sexo masculino parecem acreditar que esse planejamento é responsabilidade exclusiva da mulher, como mostra a fala da Enf2:

Os meninos não participam das atividades educativas de prevenção de gravidez. Eles não vêm a unidades, vem apenas para buscar os métodos para sua companheira que não pode vir, eles acham que é apenas mulher que participa e nem acompanham elas quando vem (ENF2).

A não participação masculina ainda é um grande problema em todas as unidades de saúde. Observando o atendimento, pode-se constatar que durante a consulta de planejamento familiar não houve participação dos adolescentes masculinos, que nem sequer estavam cadastrados na unidade de saúde para participar das atividades educativas. É necessário criar ações que possam estimular a sua participação, não só no que diz respeito à saúde sexual, mas também à saúde como um todo. Sabe-se que as mulheres procuram as unidades de saúde muito mais do que os homens, mas essa realidade deve ser mudada já na adolescencia, pois assim como a mulher, ele não só adoece, mas também tem vida sexual ativa e deve saber a maneira correta de se prevenir, saber quais são os métodos existentes e qual a sua parceira usa.

Assegurado pela Constituição Federal e também pela Lei $n^{\circ}$ 9.263, de 1996, o planejamento familiar é um conjunto de ações que auxilia as pessoas que pretendem ou não ter filhos (UNIFESP, 2011).

No Brasil, a Política Nacional de Planejamento Familiar foi criada em 2007. Ela inclui oferta de oito métodos contraceptivos gratuitos e também a venda de anticoncepcionais a preço reduzido na rede 
Farmácia Popular (BRASIL, 2007). Toda mulher em idade fértil (de 10 a 49 anos de idade) tem acesso aos anticoncepcionais nas unidades Básicas de Saúde, mas em muitos casos precisa comparecer a uma consulta prévia com profissionais de saúde. A escolha do método mais adequado deverá ser feita pela paciente, após entender os prós e contras de cada um deles.

Em 2008, o Ministério da Saúde alcançou uma marca histórica ao distribuir esses dispositivos em todos os municípios do território nacional. No ano seguinte, a política foi ampliada e houve maior acesso a vasectomias e laqueaduras, métodos definitivos de contracepção, bem como a preservativos e outros tipos de anticoncepcionais (BRASIL, 2008).

\section{CONSIDERAÇÕES FINAIS}

Com o presente trabalho foi possível observar e conhecer as estratégias desenvolvidas pelas enfermeiras da Atenção Básica quanto à prevenção da gravidez na adolescência de um município do Recôncavo da Bahia.

Em se tratando dessas estratégias, nota-se que as ações de enfermagem devem ultrapassar o atendimento na unidade, já que devem ser também ações intersetoriais. A educação em saúde não deve ocorrer apenas nas salas de espera, e sim em todos os locais que tenham público para abordar esta temática. Também é necessário ministrar palestras para os adolescentes, seus pais e seus professores.

A atuação das enfermeiras com o público adolescente ainda encontra barreiras, tornando-se necessário qualificar esses profissionais para desenvolver ações junto à família e à comunidade para consolidar os programas existentes e evitar a gravidez na adolescência.

\section{REFERÊNCIAS}

ALVES, A.; ALBINO, A. T.; ZAMPIERI, M. F. M., Um olhar das adolescentes sobre as mudanças na gravidez promovendo à saúde mental na atenção básica. Rev. Min. Enferm.;v.15, n. 4, p. 545-555, out./dez., 2011. Disponível em: < http://bases.bireme.br/cgibin/wxislind.exe/iah/online/?IsisScript=iah/iah.xis\&src=google\&base=ADOLEC\&lang=p \&nextAction=Ink\&exprSearch=617442\&indexSearch=ID>. Acesso em: 12 set. 2016.

ALMEIDA. A. C. H.;CENTA M. L. Família e a educação sexual dos filhos: implicação para a enfermagem.Act Paul enferm.V 22,N1 p 1-144 Jan/fev 2008

BRASIL (2007). Ministério da Saúde. Lei n. 8069, de 13 de julho de 1990.Disponível em: http://www.planalto.gov.br/ccivil_03/Leis/ L8069.htm. Acesso em: 10 ago. 2016.

Secretaria de Atenção à Saúde. Departamento de Ações Programáticas Estratégicas. Direitos sexuais, reprodutivos e métodos anticoncepcionais. Brasília - DF: [S.n], 2006.

Conselho Nacional de Saúde. Resolução 466/12. Disponível em: http://conselho.saude.gov.br/resolucoes/2012/Reso466.pdf. Acesso em: 20 out. 2016.

COSTA, R. F. da; QUEIROZ, M. V. O.; ZEITOUNE, R. C. G. Cuidado aos adolescentes na atenção primária: perspectivas de integralidade. Esc. Anna Nery, Rio de Janeiro,v.16, n.3, Set. 2012 . Disponível em: http://www.scielo.br/scielo.php?script=sci_arttext\&pid=S1414-81452012000300006. Acesso em: 22 ago. 2016.

FONSECA, A. L. B.; ARAÚJO, N. G. (2004). Maternidade precoce: Uma das consequências do abandono escolar e do desemprego. Rev. Brasileira de Crescimento e Desenvolvimento Humano, 14(2), 16-22. Disponível em: <www.journals.usp.br/jhgd/article/download/40001/42866>. Acesso em: 12 out. 2016.

GURGEL, M. G. I. et al. Desenvolvimento de habilidades: estratégia de promoção da saúde e prevenção da gravidez na adolescência. Rev. Gaúcha Enferm. Porto. 2008 Disponível em:

<http://www.scielo.br/pdf/rgenf/v31n 4/a05v31n4.pdf> Acesso em: 12 out. 2017. 
HIGARASHI, I.H. et al. Ações desenvolvidas pelo enfermeiro junto aos adolescentes no programa saúde da família em Maringá/paraná. Rev. Rene, Fortaleza, v.12, n.1, jan/mar, 2011.

INSTITUTO BRASILEIRO DE GEOGRAFIA E ESTATÍSTICA (IBGE). Disponível em: http://www.cidades.ibge.gov.br/xtras/perfil.php?lang=\&codmun=290980. Acesso em: 11 out. 2016.

MANFRÉ, C. C.; QUEIRÓZ, S. G.; MATTHES, A. C. S.; Considerações atuais sobre gravidez na adolescência. Rev. bras. Med. Fam. e Comum., Florianópolis, v. 5, n. 17, p. 48-54, jan./dez, 2010. Disponível em: https://www.rbmfc.org.br/rbmfc/article/viewFile/205/155. Acesso: 10 set. 2016.

MINAYO, M. C. S. O desafio do conhecimento: pesquisa qualitativa em saúde. 9. ed. São Paulo (SP): Hucitec, 2006. Acesso em: 16.09.2015

MINISTÉRIO DA SAÚDE (BR). Saúde e prevenção na escola: atitudes para curtir a vida. Brasília-DF; 2007. Disponível em:< http://www.aids.gov.br/sites/default/files/guia_forma_prof_saude_educaca o.pdf $>$. Acesso em: 18 ago. 2016.

RIBEIRO, V. C. S. et al.Papel do enfermeiro da estratégia de saúde da família na prevenção da gravidez na adolescência. Rev. Enferm. Cent. O. Min. 2016 jan/abr; 1(6):1957-1975. Disponível em:

<http://seer.ufsj.edu.br/index.php/recom/article/view/881/1006>.

SILVA, F. S.; CARVALHO, M. L. O. Enfermeiros de saúde da família e ações em planejamento familiar. UNOPAR Cient, CiêncBiol Saúde. 2007; 9(1):29-36.

SILVA,F.S.A gravidez na adolescência sob a perspectiva dos familiares:compartilhando projetos de vida e cuidado. Rev Latino-am Enfermagem 2002 março-abril; 14(2):199-206. Disponível em< http://www.scielo.br/pdf/rlae/v14n2/v14n2a08.pdf>acessado dia 03 agosto de 2017.

TABORDA, J. A. et al. Consequências da gravidez na adolescência para as meninas considerando-se as diferenças socioeconômicas entre elas. Cad. Saúde Colet., Rio de Janeiro, v. 22, n. 1, p. 16-24, 2014.

Disponível em: < http://www.scielo.br/pdf/cadsc/v22n1/1414-462X-cadsc-22-01-00016.pdf>. Acesso em: 21 ago. 2016. 